Universitas, Volumen 1, Número 2, 2008, 43-50

(C) 2008 UNAN-León, Editorial Universitaria

\title{
Prevalencia de Chlamydia Trachomatis como agente causal de Leucorrea único o en asociación con otros agentes en Mujeres Embarazadas
}

\author{
María Dolores Benavides*1; Aleyda Téllez²; Gregorio Matus ${ }^{3}$; Yubelka Susana Baltodano Martínez ${ }^{4}$, Nora \\ María Centeno Cárdenas ${ }^{4}$ \\ ${ }^{1}$ Médico Especialista en Ginecología y Obstetricia, Departamento de Ginecoobstetricia. ${ }^{2}$ MSc. en Microbiología, \\ Departamento de Microbiología. ${ }^{3}$ MSc. en Salud Pública, Departamento de Salud Pública. ${ }^{4}$ Facultad de Ciencias \\ Médicas. \\ Universidad Nacional Autónoma de Nicaragua, León (UNAN-León).
}

\section{RESUMEN}

El objetivo del presente estudio fue, identificar la prevalencia de Chlamydia Trachomatis como agente causal único o en asociación con otros agentes causales de Leucorrea en mujeres embarazadas ingresadas en el servicio de ARO del HEODRA, en el período de Agosto de 2007 a Enero de 2008.

El estudio es descriptivo, de corte transversal. Se trabajó con fuentes primarias, a través de la entrevista y exploración ginecológica de las pacientes, y secundarias, a través de la revisión de los resultados de laboratorio de las muestras tomadas. Según los resultados de la prueba rápida de detección, la prevalencia de Chlamydia Trachomatis es del 15.45\% encontrándose asociado a Gardnerella, Cándida Albicans y Tricomonas Vaginalis, no coincidiendo con los antecedentes locales en los que la prevalencia es del $20-25 \%$ y como único agente causal, pero si con la literatura extranjera consultada en los que la prevalencia en mujeres embarazadas se encuentra entre el $2-21 \%$.

Palabras Claves: Chlamydia; Prevalencia; Leucorrea.

\section{INTRODUCCIÓN}

El estudio tiene como principal objetivo, determinar la prevalencia de Chlamydia Trachomatis como agente causal único o en asociación con otros agentes causales de Leucorrea en mujeres embarazadas ingresadas en el servicio de Alto Riesgo Obstétrico (ARO) del Hospital Escuela Oscar Danilo Rosales Argüello (HEODRA) en el período comprendido de Agosto de 2007 a Enero de 2008.

La prevalencia de $C$. trachomatis en diversos estudios varía del 3 al $5 \%$ en mujeres asintomáticas. En mujeres embarazadas, se ha reportado que del 2 al $21 \%$ presentan cultivos cervicales positivos para Chlamydia.

En 1988, Elliethe G. y Col., encontraron 9\% de las mujeres estudiadas en clínica ginecológica y $12 \%$ de las mujeres en trabajo de parto, tenían infección por Chlamydia demostrable por IFD. Estudio realizado en los servicios de Ginecología y Obstetricia del Hospital Escuela de Tegucigalpa, Honduras ${ }^{[4]}$.

En 1992, Echaniz-Avilés G y Col. encontraron en el Hospital Civil de la ciudad de Morelos, México una frecuencia de $4.0 \%$ (97 pacientes) de aislamiento positivo para C. trachomatis, de éste total, un $22.66 \%$ se encontraba la Chlamydia asociada a otros agentes como

\footnotetext{
*Autores para correspondencia: benavides.dolores@yahoo.es
}

causantes de Leucorrea y en el restante porcentaje (77.34\%) se encontró la Chlamydia como único agentes causal de Leucorrea ${ }^{[5]}$.

En el 2002, Porras, F., encontró en el Servicio de Emergencia de Ginecoobstericia del HEODRA una frecuencia de $25 \%$ de Chlamydia Trachomatis como agente causal de Leucorrea ${ }^{[6]}$. En el 2003, López Aguilar, C.V., encontró en el Servicio de Emergencia de Gineco-obstetricia del HEODRA, que la Chlamydia trachomatis fue la etiología de leucorrea como único agente causal en un $16.2 \%$, y en un $9.7 \%$ en asociación con otros agentes causales, totalizando una frecuencia de aparición de $25.9 \%{ }^{[7]}$.

Pese al gran auge que están alcanzando las infecciones vulvovaginales, cervicales y uretrales en la mujer embarazada, originadas por Chlamydia Trachomatis como único agente causal o en asociación a otros microorganismos patógenos, y sus complicaciones tanto maternas como fetales, existen en nuestro medio, pocos estudios realizados para la identificación de éste microorganismo, probablemente debido al alto costo de la prueba para su detección.

Con la realización de ésta investigación se beneficia a toda la población de estudio y al personal de trabajo de ARO del HEODRA ya que se realiza el manejo específico adecuado en cada paciente en base a los resultados 
obtenidos en las pruebas de laboratorio y por ende se reduce el margen de fracaso terapéutico, motivos por los cuales llevamos a cabo el presente trabajo.

\section{DISEÑO METODOLÓGICO}

Tipo de Estudio: Descriptivo, de corte transversal.

Área de Estudio: El estudio se llevó a cabo en el servicio de ARO del HEODRA, donde se tomaron las muestras de secreción vaginal, las cuales fueron trasladadas al Departamento de Microbiología de la Facultad de Ciencias Médicas de la UNAN-León para su análisis.

Población de estudio: fueron todas las pacientes embarazadas ingresadas al servicio de ARO del HEODRA con Diagnóstico de Leucorrea.

\section{Período de Estudio: Agosto 2007 - Enero 2008.}

Fuentes de Información: Primaria, a través de la entrevista y exploración de la paciente. Secundaria, a través del reporte de los resultados de laboratorio de las muestras tomadas.

Recolección de la Información: luego de corroborar el cumplimento de los criterios de inclusión se procedió a:Toma de Muestra: A cada paciente previamente se le explicó sobre el estudio, se solicitó su aprobación para participar en él y para la toma de muestra, se le realizó un cuestionario. Se procedió a realizar el examen físico ginecológico en el cual observamos el aspecto de las secreciones, vulva, pared vaginal y cérvix.

De manera simultánea, se introdujeron 3 aplicadores de algodón estériles, se tomaron las muestras de la secreción ubicada en el fondo de saco vaginal. Uno de los aplicadores se utilizó para el frotis de tinción de gram, el otro se introdujo en un tubo que contenía medio de Stuart para cultivo posterior y el tercero se utilizó para la prueba de $\mathrm{pH}$ vaginal sobre la cinta Color $\mathrm{pH}_{\text {ast }}$.

Luego se removió el exceso de moco exocervical mediante la utilización de gasas sujetas con pinza y se introdujo un hisopo en el endocérvix hasta una altura aproximada de $2 \mathrm{~cm}$ donde se desprenden cuidadosamente células epiteliales haciendo girar el hisopo durante 10 segundos, para la detección directa del antígeno de Chlamydia Trachomatis. Las muestras fueron trasladadas al departamento de Microbiología y parasitología de la Facultad de Ciencias Médicas o al laboratorio del HEODRA según circunstancias, en un intervalo no mayor de dos horas.
La tinción de gram se utilizó para determinar alteración de la flora vaginal, búsqueda de células clave y polimorfonucleares. El examen al fresco se utilizó para la búsqueda de levaduras, Trichomona vaginalis, y prueba de aminas. El hisopo contenido en el medio de Stuart fue sometido a cultivo bacteriológico según las normas del Ministerio de Salud, incluyendo prueba de catalasa y factor de camp. Las pruebas de $\mathrm{pH}$ vaginal se realizaron de forma inmediata por quien tomó las muestras realizando frotis con el tercer hisopo sobre la cinta Color $\mathrm{pH}_{\text {ast }}$.

Las pruebas de detección de antígeno de Chlamydia Trachomatis se realizaron de forma inmediata, y los resultados fueron reportados enseguida tanto al personal médico de la sala de ARO como a la paciente.

\section{ANÁLISIS DE DATOS}

Los datos fueron procesados utilizando el programa estadístico Epi-Info y el análisis de los datos se realizó con medidas de frecuencia.

\section{Criterios de Inclusión}

- Mujeres Embarazadas.

- Que presentaran leucorrea.

- No sangrado transvaginal activo.

- Que no hubiesen usado antibióticos ni medicación vaginal en los 14 días previos.

- Que se encontraran en el período de estudio.

- Que aceptaran participar del estudio.

- Que no presentaran Amenaza de Parto Prematuro.

\section{Criterios de Exclusión}

Se excluyen del estudio a todas las mujeres que no cumplen con los criterios de inclusión.

Aspectos Éticos: Previo inicio del estudio, se introdujo el protocolo a vicerrectoría de Investigación y Posgrado de la Universidad, para su aprobación. Luego de su aprobación, se inició el estudio en el que todas las pacientes incluidas en el estudio fueron debidamente informadas de los objetivos de la investigación, a cada paciente se le pidió su consentimiento para participar en el estudio y para la toma de muestras del flujo vaginal a través de una carta firmada por ellas.

Cada paciente participante del estudio tiene conocimiento del resultado de laboratorio para su adecuado tratamiento posterior, tanto para ellas como para sus respectivas parejas. La toma de muestra se llevó a cabo en el cuarto de exploración de la sala de 
ARO del HEODRA que brindó adecuada privacidad a las pacientes, y con equipos cuya esterilidad estuvo garantizada.

Los resultados obtenidos fueron manipulados únicamente por el equipo de investigación. Los datos permanecen en confidencialidad y se utilizaron únicamente con fines investigativos. Se realizó un estudio sobre la prevalencia de Chlamydia Trachomatis como agente causal de leucorrea en mujeres embarazadas ingresadas a ARO del HEODRA en el período del 1 de Agosto de 2007 al 31 de Enero de 2008.

La población de estudio fue de 123 pacientes que según diagnóstico de ingreso presentaban Leucorrea. La mayor parte de las pacientes de nuestra población estuvo comprendida en el grupo etáreo de 20-35 años con un $55.3 \%$, seguida de las menores de 20 años con un $35.8 \%$ (Figura 1 ).
Respecto al nivel de escolaridad el $45.53 \%$ de las pacientes tenían estudios primarios, seguidas de pacientes con estudios secundarios con $29.27 \%$ y universitarios con $19.51 \%$ (Figura 2). El estado civil de la mayor parte de la población fue "Unión libre" con $66.67 \%$, seguida de las Casadas con $27.64 \%$ y las Solteras con $5.69 \%$ (Figura 3). De los antecedentes obstétricos de las pacientes, podemos mencionar, que el $43.9 \%$ de ellas eran Primigestas, seguidas de un $29.27 \%$ de pacientes Bigestas, un $17.07 \%$ de Trigestas y $9.76 \%$ de pacientes eran Multigestas (Tabla 1 ).

Respecto a la Paridad el $51.9 \%$ eran pacientes Nulíparas y $39.4 \%$ Multíparas (Tabla 2). El $7.32 \%$ de las pacientes tenía antecedentes de Aborto y $17.89 \%$ antecedentes de Cesárea. El $91.87 \%$ de las pacientes incluidas, fueron pacientes ingresadas a la sala de ARO II (Figura 4). El 67.48\% de las pacientes refirieron haber tenido solamente un compañero sexual, y un $22.76 \% 2$ compañeros (Figura5).

Figura 1. Edad de las pacientes ingresadas a la sala de ARO del HEODRA con diagnóstico de Leucorrea en el período de estudio.

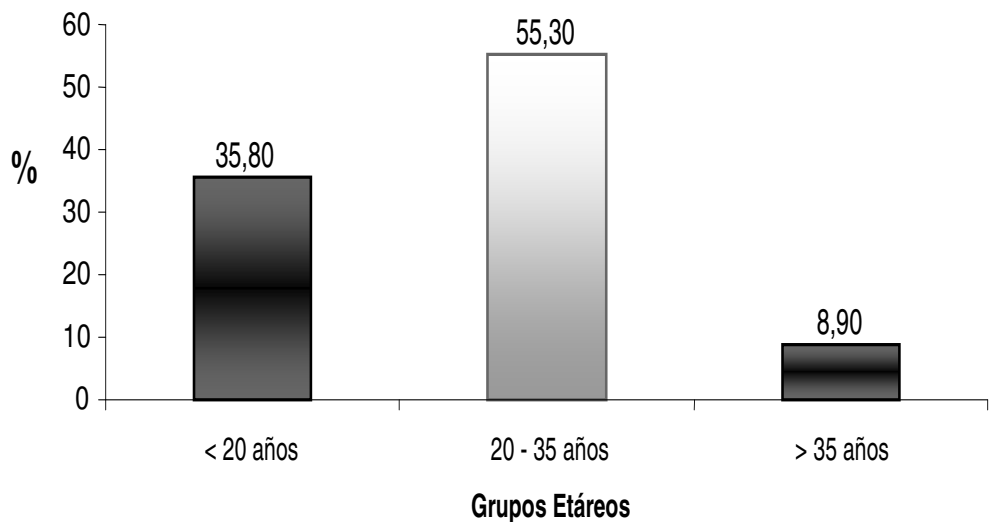

Figura 2. Nivel de escolaridad de las pacientes ingresadas a la sala de ARO del HEODRA con diagnóstico de Leucorrea en el período de estudio.

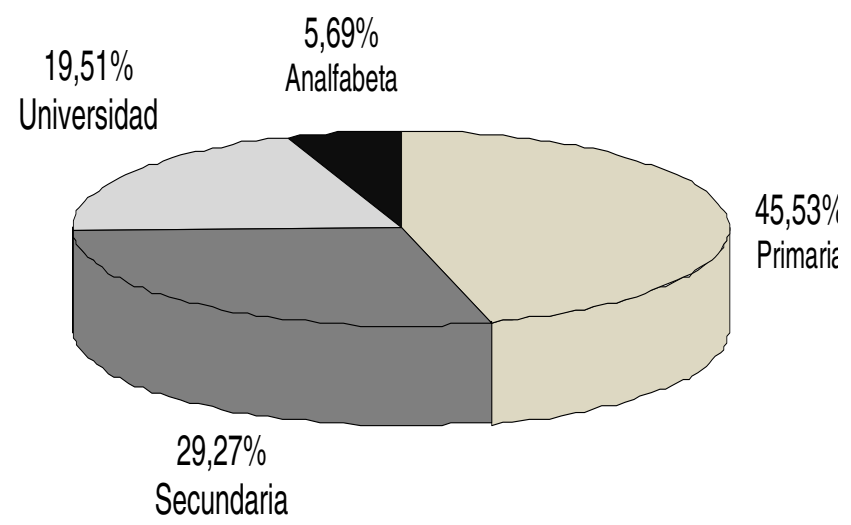


Figura 3. Estado civil de las pacientes ingresadas a la sala de ARO del HEODRA con diagnóstico de Leucorrea en el período de estudio.

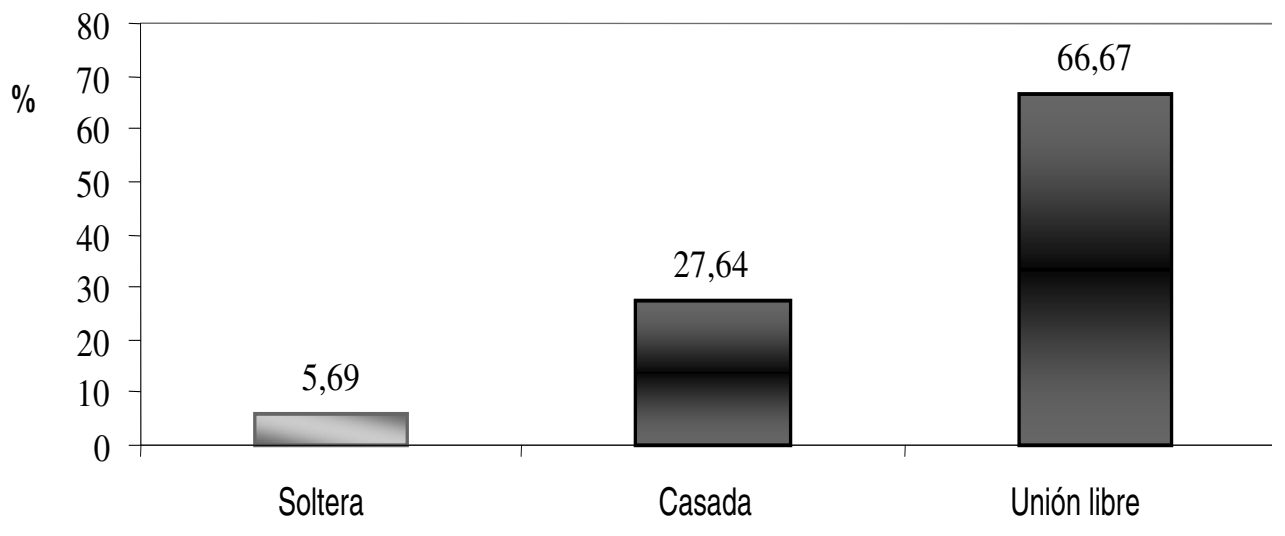

Estado Civil

De acuerdo a lo referido por las pacientes, el 53,65\% tuvo su última relación sexual de 1 semana a 1 mes previo a su ingreso, y un $37,4 \%$ hace más de 1 mes (Figura 6). El 58.54\% de las pacientes del estudio no utilizaban ningún método de planificación previo a su embarazo, y un $21.14 \%$ utilizaba métodos hormonales (Figura 7).

Tabla 1: Gestación de las pacientes ingresadas a ARO del HEODRA con diagnóstico de Leucorrea en el período de estudio

\begin{tabular}{|c|c|c|c|c|c|}
\hline & $\begin{array}{c}\text { Primi } \\
\text { Gestas }\end{array}$ & $\begin{array}{c}\text { Bi } \\
\text { Gestas }\end{array}$ & $\begin{array}{c}\text { Tri } \\
\text { Gestas }\end{array}$ & $\begin{array}{c}\text { Multi } \\
\text { Gestas }\end{array}$ & $\begin{array}{c}\text { Total } \\
\text { Gestas }\end{array}$ \\
\hline Frecuencia & 54 & 36 & 21 & 12 & 123 \\
\hline Porcentaje & $43.90 \%$ & $29.27 \%$ & $17.07 \%$ & $9.76 \%$ & $100 \%$ \\
\hline
\end{tabular}

En los antecedentes patológicos personales de las pacientes, el de mayor predominio fue Leucorrea, referido por el $100 \%$ de ellas, seguido de Infecciones de Vías Urinarias con un $30.89 \%$ y Diabetes Mellitas con un $9.76 \%$ (Tabla 3 ).

Tabla 2: Paridad de las pacientes ingresadas a ARO del HEODRA con diagnóstico de Leucorrea en el período de estudio.

\begin{tabular}{|c|c|c|c|c|}
\hline & Paridad & Nuliparas & Multíparas & Gran Multioparas \\
\hline Frecuencia & 104 & 54 & 41 & 9 \\
\hline Porcentaje & $100 \%$ & $51.90 \%$ & $39.40 \%$ & $8.70 \%$ \\
\hline
\end{tabular}

Figura 4. Semanas de Amenorrea de las pacientes ingresadas a la sala de ARO del HEODRA con diagnóstico de Leucorrea en el período de estudio.

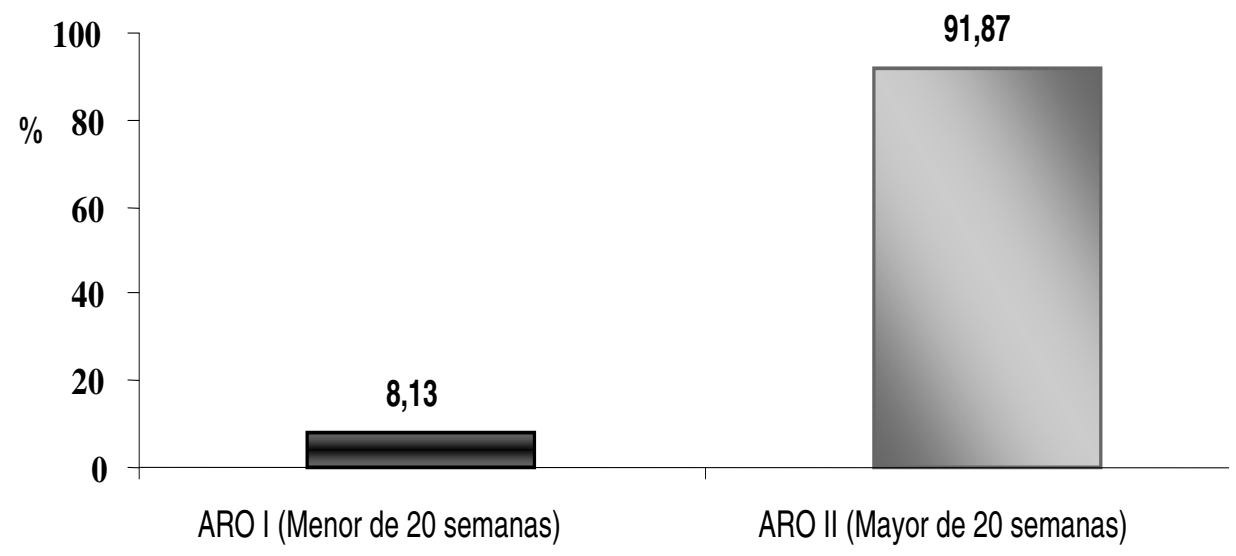

Semanas de Amenorrea 
El $79.67 \%$ de las Secreciones vaginales de las pacientes fue de color Blanquecino, $100 \%$ de ellas era de tipo Mucoso y $79.67 \%$ no era Fétido. Además en el examen físico de las pacientes se encontró que el $13.01 \%$ de ellas presentaba edema al momento de la inspección. El $74.8 \%$ de las pacientes de nuestro estudio presentó un $\mathrm{pH}$ vaginal menor de 4.6 , y el restante $25.2 \%$ un $\mathrm{pH}$ mayor de 4.6 (Figura 8). La prevalencia de Chlamydia Trachomatis, fue de un $16.24 \%$ de las pacientes en estudio, en las que las pruebas rápidas de detección del agente resultaron positivas.
Tabla 3. Antecedentes Patológicos de la Pacientes ingresadas a ARO del HEODRA con diagnóstico de Leucorrea en el período de estudio.

\begin{tabular}{|c|c|}
\hline Patologías & Porcentajes \\
\hline Leucorrea & $100 \%$ \\
\hline IVU & $30.89 \%$ \\
\hline Diabetes & $9.76 \%$ \\
\hline Amenaza Parto Prematuro & $5.69 \%$ \\
\hline Amenaza de Aborto & $6.50 \%$ \\
\hline
\end{tabular}

Figura 5. Número de compañeros sexuales de las pacientes ingresadas a la sala de ARO del HEODRA con diagnóstico de Leucorrea en el período de estudio.

\section{$9,76 \%$}

Tres o más

compañeros

\section{$22,76 \%$ \\ Dos o más \\ compañeros}

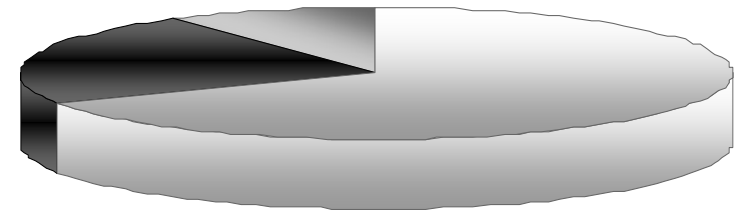

$67,48 \%$

Un compañerc

Figura 6. Período transcurrido desde la última relación sexual de las pacientes ingresadas a la sala de ARO del HEODRA con diagnóstico de Leucorrea en el período de estudio.

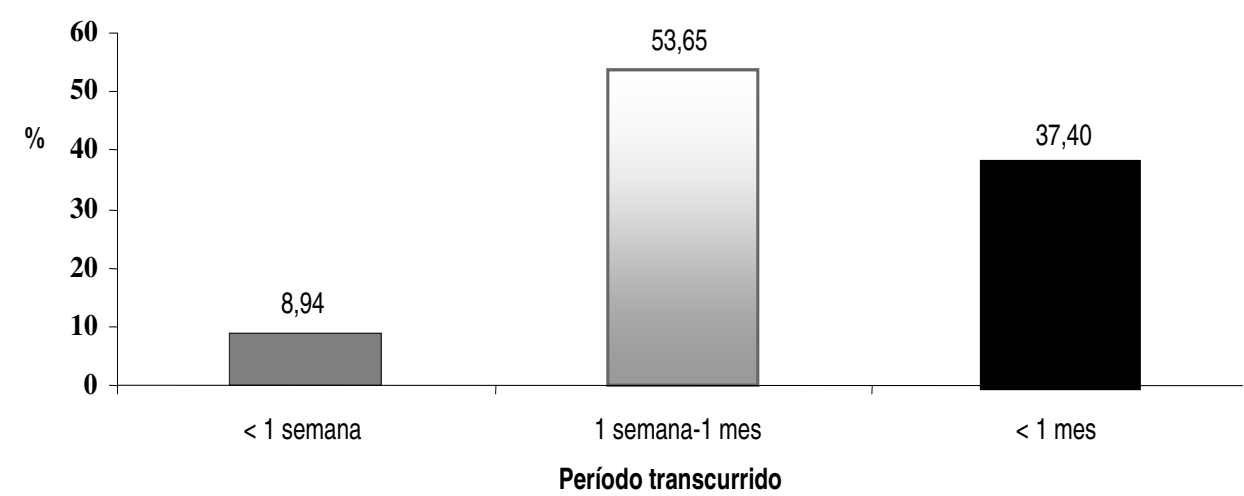

Figura 7. Métodos de planificación familiar de las pacientes ingresadas a la sala de ARO del HEODRA con diagnóstico de Leucorrea en el período de estudio.

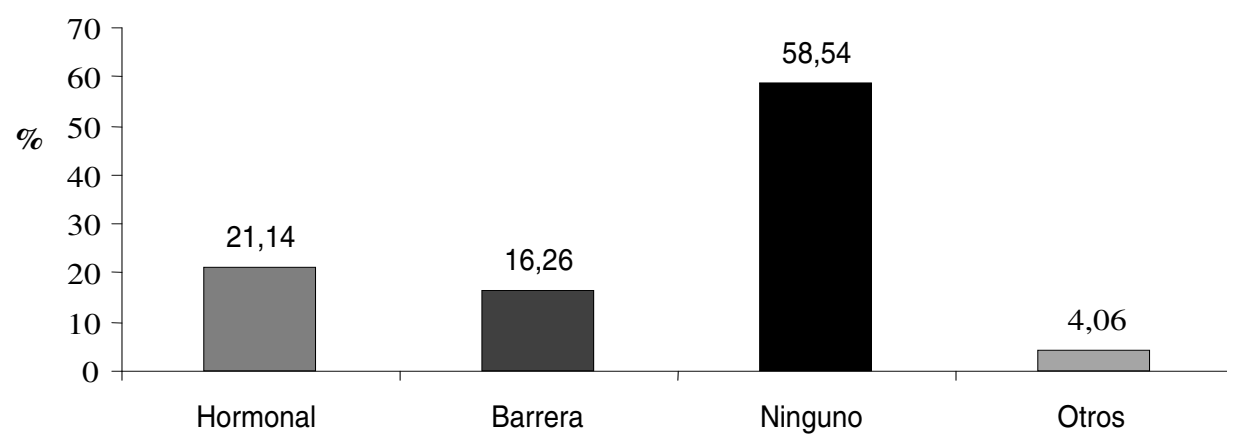

Métodos de Planificación 
En las pacientes que se encontró positiva la prueba rápida de detección de Chlamydia Trachomatis, se encontró también Gardnerella como principal microorganismo asociado causante de Leucorrea.

\section{DISCUSIÓN DE LOS RESULTADOS}

Muestran los resultados obtenidos que las adultas jóvenes así como las de bajo nivel socioeconómico, relacionadas con un bajo nivel cultural, presentan un mayor riesgo para la adquisición de enfermedades que afectan el tracto genital inferior, muchas de las cuales se transmiten sexualmente, por lo que también desempeñan un papel importante el inicio de la vida sexualmente activa y el número de compañeros sexuales.

Aunque según, los resultados encontrados en nuestro estudio, la mayoría de las pacientes refirió haber tenido solo un compañero sexual, se debe considerar en nuestra cultura machista, la promiscuidad que pudiera tener el compañero sexual y el hecho que la mayoría de pacientes no dicen en realidad cuantos compañeros sexuales han tenido.

Tabla 4.Características de secreción vaginal de las pacientes ingresadas a ARO del HEODRA con diagnóstico de Leucorrea en el período de estudio.

\begin{tabular}{|l|l|c|c|}
\hline & \multicolumn{1}{|c|}{ Característica } & Frecuencia & Porcentaje \\
\hline \multirow{4}{*}{ Color } & Blanquecina & 98 & $79.67 \%$ \\
\cline { 2 - 4 } & Amarilla-Verdosa & 13 & $10.57 \%$ \\
\cline { 2 - 4 } & Gisácea & 12 & $9.76 \%$ \\
\hline \multirow{3}{*}{ Tipo } & Mucosa & 123 & $100 \%$ \\
\cline { 2 - 4 } & Mucopurulenta & 0 & $0 \%$ \\
\hline \multirow{3}{*}{ Olor } & Fétida & 15 & $12.20 \%$ \\
\cline { 2 - 4 } & Fétida (olor a pescado) & 10 & $8.13 \%$ \\
\cline { 2 - 4 } & No fétida & 98 & $79.67 \%$ \\
\hline
\end{tabular}

Figura 8: $\mathrm{pH}$ vaginal de las pacientes ingresadas a la sala de ARO del HEODRA con diagnóstico de Leucorrea en el período de estudio.

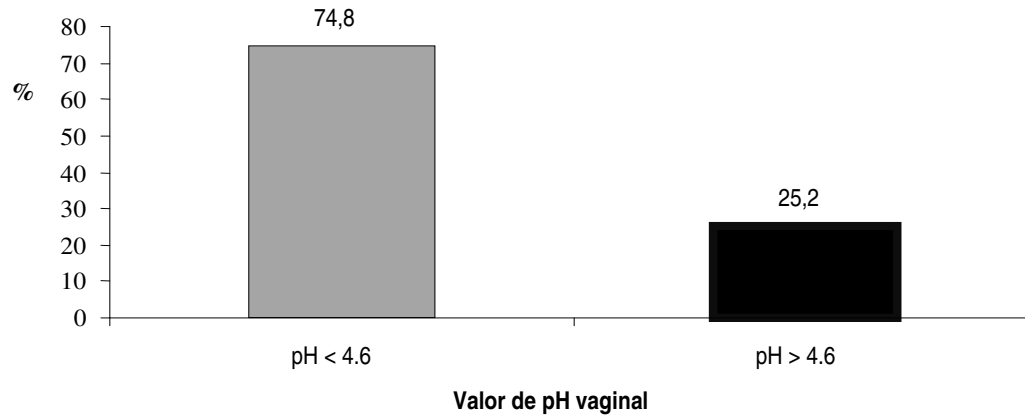

Figura 9. Prevalencia de Chlamidia Trachomatis como agente causal de Leucorrea en las pacientes ingresadas a la sala de ARO del HEODRA con diagnóstico de Leucorrea en el período de estudio.

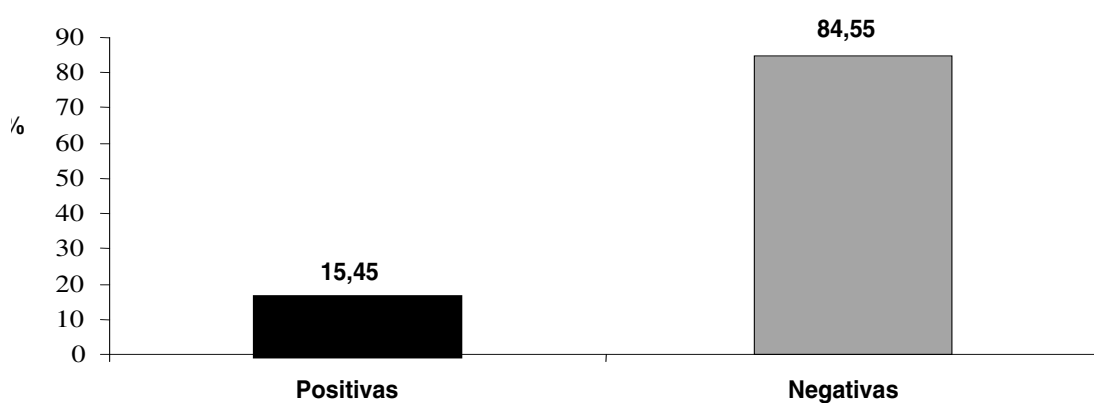


Del total de las pacientes incluidas en el estudio la gran mayoría de las pacientes corresponden a ingresos a la sala de ARO II, lo que coincide con lo esperado ya que gran porcentaje de ingresos a la sala de ARO I son debidos a Amenazas de Aborto o Abortos, por lo que quedaron excluidas de nuestra investigación.

La mayoría de las pacientes de nuestro estudio tuvieron su última relación sexual en un período menor o igual a 1 mes, lo que explicaría la leucorrea por Chlamydia ya que el período de incubación de ésta es de 10-21 días en la mayoría de las literaturas consultadas.

Con respecto a los métodos de planificación, observamos en los resultados obtenidos en el estudio, que el porcentaje de pacientes que utilizan métodos de barrera es relativamente bajo, lo que se puede atribuir al aspecto cultural, ya que en nuestro país la cultura machista influye en la no utilización de éstos métodos, que son protectores para evitar las Infecciones de Transmisión Sexual.

De los antecedentes patológicos personales, merece mención el antecedente de Leucorrea en $100 \%$ de las pacientes, la cual es el principal síntoma de vaginitis; seguido de las infecciones de Vías Urinarias, las que por contigüidad pueden afectar el tracto genital; otra patología a mencionar, es la Diabetes, en la que se produce un cambio en el $\mathrm{pH}$ vaginal y puede alterar la flora vaginal. La Amenaza de Parto Prematuro y Amenaza de Aborto tienen como causa etiológica la presencia de infecciones vaginales, que se puede relacionar al hecho de que el cien por ciento de nuestras pacientes tienen antecedente de Leucorreas que pudieron complicar embarazos previos.

De acuerdo, a las características de la secreción vaginal, encontramos coincidencia con las literaturas consultadas, ya que $79.67 \%$ de ellas fueron de color blanquecino, $79.67 \%$ eran no fétidas y $100 \%$ de ellas eran mucosas, y la literatura refiere que la infección por éste microorganismo generalmente es asintomática.

La infección por Chlamydia Trachomatis es de distribución mundial y su importancia como problema de salud pública depende de la región geográfica estudiada. Tanto en los países industrializados como en los países en desarrollo es en la actualidad la infección de transmisión sexual más frecuente entre las mujeres en edad fértil, se estima que en cualquier lugar geográfico, nivel socioeconómico o raza la prevalencia de la infección es de por lo menos 5\%.
Los resultados revelados en nuestro estudio presentan una prevalencia de Chlamydia Trachomatis de 15.45\% (Figura 9), lo que no coincide con los estudios previos consultados, como las tesis elaboradas por el Dr. Porras y la Dra. C. López, quienes manifiestan una prevalencia en sus estudios entre el $20-25 \%$, pero que sí coincide con literaturas internacionales consultadas, ya que se encuentra dentro del rango.

Respecto al pH vaginal, los resultados encontrados en nuestro estudio, coinciden con la literatura consultada ya que el total de pacientes Chlamydia positivas presentaron un $\mathrm{pH}$ superior a 4.6, y la literatura refiere que tanto la Chlamydia como la Gardnerella y la Tricomona (algunas asociaciones encontradas), se desarrollan en pH más alcalinos.

En cuanto a la asociación de la Chlamydia Trachomatis con otros agentes en nuestro estudio, encontramos como principal agente asociado a la Gardnerella, que según las literaturas consultadas el segundo agente más frecuente que se asocia con Chlamydia, a la vez encontramos en las mismas pacientes, la asociación con lesiones condilomatosas que ratifica el hecho de que $C$. Trachomatis es un microorganismo de transmisión sexual. Otras asociaciones que se encontraron fueron Cándida Albicans y Tricomonas Vaginalis.

\section{CONCLUSIONES}

La población de estudio sé caracterizó por ser adultos jóvenes, con estudios primarios, con estado civil predominante de unión libre, primigestas, nulíparas, con más de 20 semanas de amenorrea, sin utilizar métodos de planificación y que han tenido solo un compañero sexual.

La prevalencia de Chlamydia Trachomatis como único agente causal, no se presentó, su prevalencia en asociación con otros agentes fue de $16.24 \%$, casos en los que se encontró junto a la presencia de Gardnerella, Cándida Albicans y Tricomonas Vaginalis.

La única característica clínica encontrada en pacientes Chlamydia positivas fue el edema del cérvix.

Las características de la secreción vaginal de las pacientes Chlamydia positivas, asociadas a Gardnerella, fueron secreciones grisáceas y amarillo-verdosas, coincidiendo parcialmente con las características de secreción de infección por estos microorganismos, las de las pacientes con Cándida y Tricomonas asociadas a Chlamydia si coincidieron totalmente, al ser secreciones blanquecina y amarillo-verdosa, respectivamente. 


\section{AGRADECIMIENTO}

- Al Programa de Pequeñas Ayudas para Investigación (PAI), por financiar la investigación.

- Al Laboratorio de Microbiología de la Facultad de Ciencias Médicas y al Laboratorio del HEODRA, por el procesamiento de las muestras.

- Al personal de la Sala de ARO del HEODRA y a las pacientes ingresadas en éste servicio por su colaboración y participación en el estudio.

\section{BIBLIOGRAFÍA}

1) Botero $U$, Jaime, Jubiz $H$, Alfonso, Henao, Guillermo Henao. 2004. Obstetricia y Ginecología. 7ma Edición. Editorial CIB. Pp. $72-76$

2) Schwarcz Ricardo Leopoldo, Diverges Carlos Alberto. 2003. Obstetricia. Quinta Edición. Editorial. El Ateneo.

3) Faro Sebastián, Soper David. 2002. Enfermedades Infecciosas de la Mujer. 1ra Edición. Editorial Mc Graw Hill-Interamericana. Cap. 2. Pp. 44-58. Cap.20. Pp. 260266. Pp. 486-499.

4) E. Girón, G. Vallecillo, C.A. Javier Zepeda. Infecciones por Chlamydia Trachomatis Ginecoobstétricas del Hospital Escuela Servicios de Ginecoobstetricia y Microbiología Clínica. H.E. Tegucigalpa. Revista Médica Hondureña. Vol. 57-1989.

5) Echániz Avilés, G. Calderón Jaime E. (1992). Prevalencia de infección cervicovaginal por Chlamydia Trachomatis en población femenina embarazada en la ciudad de Cuernavaca, Morelos. Salud pública mexicana 1992. Mayo - Junio de 1992. Vol. 31 Nº 3.

6) Dr. Porras Díaz Felipe. Infección cervical por Chlamydia Trachomatis en mujeres embarazadas. HEODRA-León. Noviembre - Diciembre 2002. Tesis UNAN León 2002.

7) Dra. López Aguilar Claudia. Agentes etiológicos más frecuentes de infección cervicovaginal en mujeres embarazadas atendidas en el HEODRA, León de octubre a diciembre del 2003. Tesis UNAN León 2003.

8) La leucorrea y su significado clínico. www.drscope.com/privados/pac/generales/ginecologia/ leucorrea. consultada01/05/07 a las 10:00am.

9) Brooks Geo. Batel Janet. Microbiología Médica de Jawetz, Melnick y Adelberg. 17ma edición. Editorial El Manual Moderno.2002 . Cap. 15 Pp 385- 395.
10) Pérez Sánchez A. y Donoso Siña E. Obstetricia. Segunda edición, Mediterráneo, Santiago Chile. 1992. Cap. 46 .Pp $693-701$.

11) Jones $H$ w. Coltons AC. Burnett. Tratado de Ginecología de Novak. 13 va edición. Editorial Interamericana. 2002.Cap. 15. Pp. 371-382.

12)Pernoll M. Benson R. Diagnóstico y Tratamiento Ginecoobstétrico. Quinta Edición. Editorial El Manual Moderno. México DF. 1998. Pp.629-640.

13) OMS. Diagnóstico de Laboratorio de las Enfermedades de Transmisión Sexual.2000. Cap. 2. Pp.22-34.

14) OMS. Infecciones de Transmisión Sexual y Otras Infecciones del Tracto reproductivo. 2005. Pp. 115, 143145, 193. 